\title{
Mesoporous silica hollow capsules embedded with magnetic nanoparticles
}

\author{
S. Yoshikawa, N. Kato*, and G. Obara \\ Graduate School of Science and Technology, Meiji University, Kawasaki 214-8571, Japan
}

\begin{abstract}
Our method for synthesizing the mesoporous silica hollow capsules (MSHCs) was combined with the Layer-by-Layer method to integrate the magnetic nanoparticles (mNPs) onto the core template for the MSHC synthesis. The resultant magnetic MSHCs (mMSHCs) were monodisperse as the conventional MSHCs, and the mNPs were embedded on the inner surface of the mesoporous silica shell. The same mesoporous structure as in the MSHCs was confirmed in the mMSHCs and the properties of the mNPs were maintained even after being incorporated into the mMSHCs.
\end{abstract}

\section{Introduction}

The mesoporous silica hollow capsules (MSHCs) have great potential as drug carriers in drug delivery system, carriers of enzyme or catalysis, and adsorption and separation materials. To produce the MSHCs, numbers of methods based on the templated-assisted strategy have been proposed $[1,2]$. We had also reported the methods for synthesizing the monodisperse MSHCs with uniform shell thickness $[3,4]$, and the loading and release of dyes had been demonstrated [3, 5]. In our methods, the surfactants and polystyrene (PS) nanoparticle were used as the templates to form the mesoporous structure in the shell and the hollow cavity, respectively.

In addition to the inherent feature of MSHCs, i.e., the large hollow cavity and the mesoporous network in the shell, further functionalized MSHCs such as luminescent and/or magnetic MSHCs (mMSHCs) are of interest for theranostic purposes $[1,6]$. Specifically, the magnetic properties allow the magnetically-guided drug delivery for the therapy and the contrast-enhanced magnetic resonance imaging for the diagnosis. So far, various types of mMSHCs have been reported; the mMSHC incorporating the mNPs on the outer surface of the shell [7], inside the shell $[8,9]$, or in the hollow cavity [10-12] and the rattle-type mMSHC that encapsulates one magnetic particle in its hollow cavity [13-18].

Here, we aim to extend our method to produce the mMSHC that has the same uniformities in size and shape and the same mesoporous structure compared to the MSHCs reported before [3]. To incorporate the mNPs, the Layer-by-Layer (LbL) method [19] that had provided the magnetic hollow microspheres $[20,21]$ has been combined with our method, i.e., the multilayers of polyelectrolytes and mNPs were formed on the PS particle via the LbL method, followed by the sol-gel reaction to form the layer of silica/surfactant complex on the LbL layer. The coated particles were then calcined to pyrolyze the PS and surfactants, resulting in the mMSHCs.

\section{Materials and methods}

The mNPs were prepared based on the chemical coprecipitation mothed reported in the literature [22]. 100 $\mathrm{mL}$ of solution containing $17.4 \mathrm{mM} \mathrm{FeCl}_{3}$ and $8.4 \mathrm{mM}$ $\mathrm{FeCl}_{2}$ was made using an aqueous $\mathrm{HCl}(0.2 \mathrm{M})$ as a solvent, followed by the addition of $5.4 \mathrm{~mL}$ of an aqueous $\mathrm{NaOH}(10 \mathrm{M})$. The solution was stirred at R.T. for $1 \mathrm{~h}$ and at $90{ }^{\circ} \mathrm{C}$ for another $1 \mathrm{~h}$. With the aid of a magnetic separation, the mNPs were collected and redispersed in 6 $\mathrm{mL}$ of an aqueous sulfuric acid $(9.5 \% \mathrm{w} / \mathrm{w})$, followed by the a stirring at R.T. for $5 \mathrm{~min}$ to reduce the size of mNPs. By the magnetic separation, the mNPs were washed with water until the $\mathrm{pH}$ became neutral and redispersed in 100 $\mathrm{mL}$ of aqueous citric acid $(0.3 \mathrm{M})$, followed by a stirring at $90{ }^{\circ} \mathrm{C}$ for $30 \mathrm{~min}$. The dispersion medium was then exchanged to ethanol, and the sample was dried. All the processes were carried out under $\mathrm{N}_{2}$ atmosphere.

The mNPs, polyethylenimine (PEI, Mw 25 000), and poly(diallyl dimethyl ammonium chloride) (PDDA, $\mathrm{Mw}>100$ 000) were used to form the LbL assembly on the PS particles dispersed in water $(5 \% \mathrm{w} / \mathrm{v}$, microParticles $\mathrm{GmbH})$. Aqueous solutions of PEI and PDDA $(1 \mathrm{mg} / \mathrm{mL})$ and an aqueous dispersion of mNPs $(20 \mathrm{mg} / \mathrm{mL})$ were prepared as the adsorption solutions. $137 \mu \mathrm{L}$ of PS dispersion was diluted by pure water. 1) The dispersion was centrifuged, the supernatant was removed, and the sediment was dispersed by sonication. 2) To build a layer, $1.5 \mathrm{~mL}$ of the adsorption solution was

\footnotetext{
* Corresponding author: nkato@meiji.ac.jp
} 
added and the dispersion was incubated for $20 \mathrm{~min}$ on the vortex mixer to let the material adsorb on the particles. 3) After the incubation, the dispersion medium was exchanged to pure water to remove the excess material by using a centrifuge. The procedures from 1) to 3) were repeated by exchanging the adsorption solutions in accordance with the order of the layer structure (PS particle/PEI/mNPs/PDDA). Finally, the coated particles were dispersed in $1 \mathrm{~mL}$ of pure water.

After stirring the mixture of the dispersion of the coated particles $(1 \mathrm{~mL})$, cetyltrimethylammonium bromide $(50 \mu \mathrm{mol}), \mathrm{H}_{2} \mathrm{O}(11 \mathrm{~mL})$, ethanol $(4 \mathrm{~mL})$, and aqueous $\mathrm{NH}_{3}(25 \% \mathrm{w} / \mathrm{w}, 0.1 \mathrm{~mL})$ for $10 \mathrm{~min}$ at $30{ }^{\circ} \mathrm{C}$ under $\mathrm{N}_{2}$ atmosphere, tetraethoxysilane $(50 \mu \mathrm{L})$ was added to initiate the sol-gel reaction and form the silica/surfactant complex on the coated particles, and the stirring was carried out for another $2 \mathrm{~h}$ at $30{ }^{\circ} \mathrm{C}$. The dispersion medium was then exchanged to ethanol by using a centrifuge. The sample was dried and calcined at $550{ }^{\circ} \mathrm{C}$ for $6 \mathrm{~h}$ in ambient atmosphere to remove the templates.

Pure water $(>18 \mathrm{M} \Omega \mathrm{cm}$ ) used for the mNP synthesis, the $\mathrm{LbL}$ coating and the sol-gel reaction was prepared in a Milli-Q system and degassed prior to use.

The $\zeta$-potential and the diameter of the particles were evaluated by laser Doppler electrophoresis and dynamical light scattering (DLS) methods, respectively (Otsuka Electronics, ELSZ). The Hückel equation was used to obtain the values of $\zeta$-potential [23], and the Marquardt method [24] was applied to obtain the distribution of scattered light intensity versus particle diameter. The morphology of the samples was observed using the transmission electron microscope (TEM, JEOL, JEM$1200 \mathrm{EXII})$ by placing the dispersion on the $\mathrm{Cu}$ grid covered by collodion film (Nisshin EM). X-ray diffraction (XRD) patterns were recorded on the $\theta-2 \theta$ powder diffractometer (Rigaku, ULTIMA III) using $\mathrm{Cu}$ $\mathrm{K} \alpha 1$ radiation. The crystallite sizes were calculated by applying the Scherrer equation to the diffraction peak [25]. The magnetization curves of the samples were recorded on a vibrating sample magnetometer (TOEI, TEM-WF86R-154). The saturation magnetization (Ms) and coercivity field values (Hc) were obtained from the magnetization curves.

\section{Results and discussion}

\subsection{Fabrication of $\mathrm{mMSHCs}$}

The $\zeta$-potential was observed after each coating as shown in Figure 1. When the first polycation (PEI) layer was formed on the negatively charged bare PS particles, the sign of the potential was reversed. Because the $\zeta$-potential of the mNPs was $-22.6 \mathrm{mV}$, the sign reversal from positive to negative was observed upon formation of the second mNP layer. Such sign reversal was also observed after coating the third polycation (PDDA) layer. This result suggests that the charge overcompensation occurred upon the adsorption of each layer.

The morphologies of the particles were observed by the TEM. Figure 2(a) shows the bare PS particles and their average dimeter was $202.5 \mathrm{~nm}$. As shown in Figure 2(b), the small black dots densely adsorbed on the smooth surface of the bare particles were observed. Because the black dots were observed after the formation of the $\mathrm{PEI} / \mathrm{mNP} / \mathrm{PDDA}$ layer on the particles, these dots corresponded to the mNPs and the average size of the dots in Figure 2(b) was $10.4 \mathrm{~nm}$. These results indicate that the LbL assembly on the PS particles was successfully achieved.

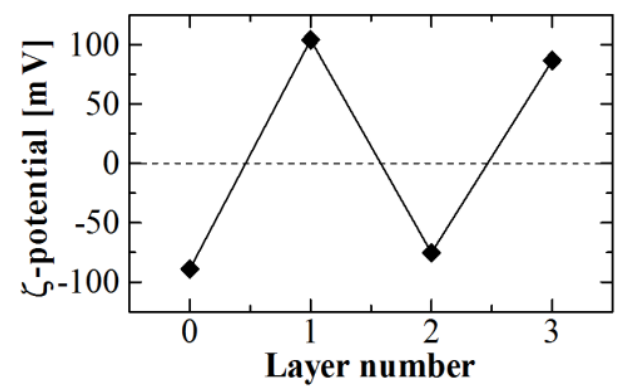

Fig. 1. $\zeta$-potential as a function of layer number. The second layer was composed by the mNPs.

On the particles with the LbL coating, the silica/surfactant complex was formed and the sample was calcined to remove the templates. The TEM image after the calcination is shown in Figure 2(c). The image clearly shows the complete removal of the PS core and the formation of the hollow structure. On the inner surface of the cavity, many black dots, which were similar to the ones observed on the coated particles in Figure 2(b), were observed. Therefore, the mNPs were remained even after the calcination and embedded on the inner surface of the capsules, and the formation of mMSHCs was suggested.

The comparison between mMSHCs and the conventional MSHCs was performed. The MSHCs were prepared under the same condition for the mMSHC synthesis except the use of the PS particles without any coatings. The TEM image of the MSHCs shown in Figure 2(d) exhibits the same morphology as in the previous reports [3-5]. The mMSHCs also show the same shell thickness and the smooth outer surface as shown in Figure 2(c). However, the inner surface of the mMSHCs is not as smooth as that of the MSHCs. This is because the former capsules embedded the mNPs on the inner surface of the cavity. The outer and inner diameters of the mMSHCs and the MSHCs, which were determined by the TEM images, are listed in Table 1. The both diameters of the mMSHCs are larger than those of the MSHCs, indicating that the diameter of the core template for the mMSHCs was larger due to the LbL coating.

The particle size distributions of the bare PS particles and the mMSHCs are also compared in Figures 3(a) and (b). From the distribution, the coefficient of variation (CV) of diameter was calculated, and the values of CV were $26.3 \%$ and $43.4 \%$ in the bare particles and mMSHCs, respectively. The increase in the $\mathrm{CV}$ was comparable to the MSHCs [3, 4]. The structure of the mesopores in the shell was also examined by the XRD. As shown in Figure 4, the mMSHCs exhibited a broad peak at $2 \theta=2.56^{\circ}$. Although these characteristics indicated that the periodicity of the mesopores was highly disordered, the same XRD patterns were observed in the 
MSHCs [3, 4]. Therefore, not only the incorporation of mNPs in the capsules but also the same structural characteristics as in MSHCs was realized in the obtained mMSHCs.
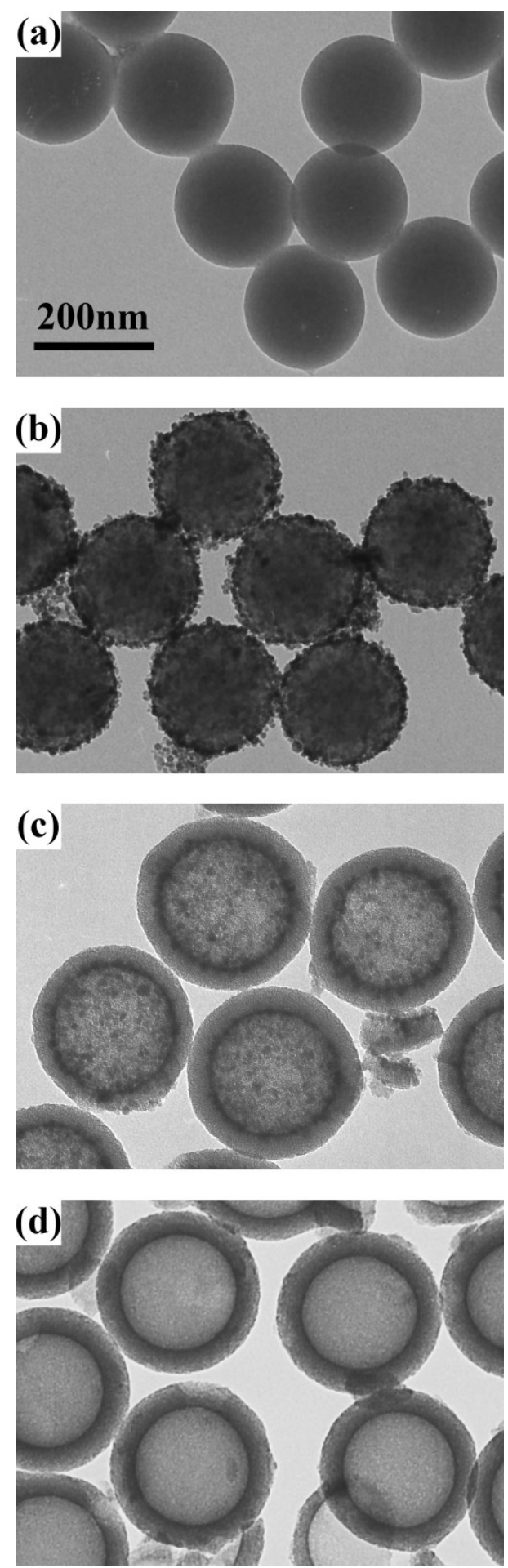

Fig. 2. TEM images of (a) bare PS particles, (b) particles coated by the layer structure of $\mathrm{PEI} / \mathrm{mNP} / \mathrm{PDDA}$, (c) mMSHCs, and (d) MSHCs. A scale bar for all images is indicated in (a).

Table 1. Sizes of mMSHCs and MSHCs analysed by TEM.

\begin{tabular}{ccc}
\hline Sample & Outer diameter & Inner diameter \\
\hline mMSHCs & $284.9 \mathrm{~nm}$ & $210.8 \mathrm{~nm}$ \\
MSHCs & $264.4 \mathrm{~nm}$ & $190.8 \mathrm{~nm}$ \\
\hline
\end{tabular}

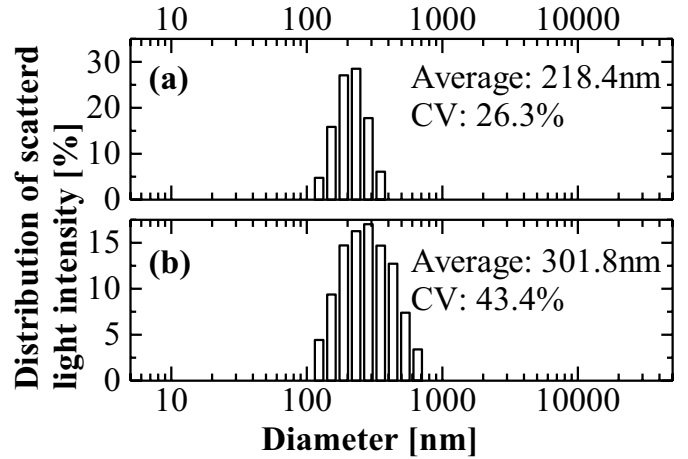

Fig. 3. Particle size distributions of (a) bare PS particles and (b) mMSHCs. Average diameters and CV values were indicated.

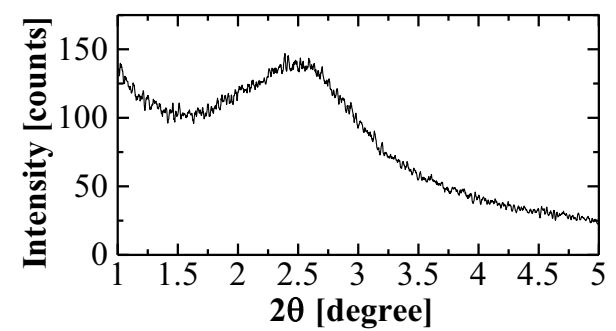

Fig. 4. XRD pattern of the mMSHCs scanned at low $2 \theta$.

\subsection{Comparison between mNPs and mMSHCs}

The XRD pattern of the as-prepared mNPs shown in Figure 5 indicated that our mNPs consisted of magnetite $\left(\mathrm{Fe}_{3} \mathrm{O}_{4}\right)$ crystallites. By applying the Scherrer equation to the (400) diffraction peak, the size of the crystallites was calculated to be $7.8 \mathrm{~nm}$. The mNPs in the mMSHCs also exhibited the same XRD pattern as observed in the mNPs except a broad peak around $2 \theta=24^{\circ}$. This peak is the halo pattern of $\mathrm{SiO}_{2}$ consisting of the shell. By applying the Scherrer equation, the size of the crystallites embedded in the capsules was deduced to be $7.5 \mathrm{~nm}$. Therefore, the magnetite crystallites maintained their size even after the sol-gel reaction and the calcination to form the hollow capsules. The size of the crystallites is comparable to the size of the black dots (ca. $10.4 \mathrm{~nm}$ ) observed in the TEM images as shown in Figures 2(b) and (c). This similarity in size also supports that the black dots in the TME images correspond to the MNPs.

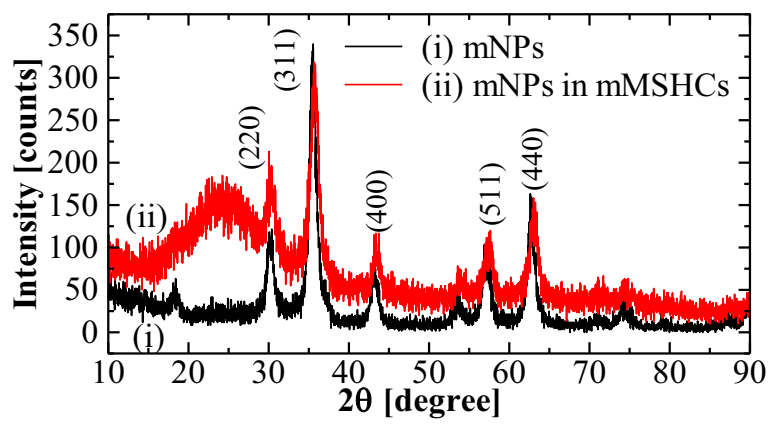

Fig. 5. XRD patterns of (i) mNPs and (ii) mMSHCs.

The magnetization curves of the mNPs and mMSHCs are indicated in Figure 6(a). The Ms values of mNPs and mMSHCs were 57.9 and $14.9 \mathrm{emu} / \mathrm{g}$, respectively. Because the mass ratio of mNPs was lower in the mMSHCs, the reduction of Ms was observed after the 
formation of mMSHCs. However, our preparation method of the mMSHCs provided the Ms value comparable to the reported magnetic capsules [8-18]. As shown in Figure 6(b), the Ms value of our mMSHCs was large enough to show a magnetic separation using a neodymium magnet. The inset of Figure 6(a) shows that mNPs and mMSHCs exhibited the Hc of 25.4 and 52.1 Oe, respectively, indicating that our magnetite crystallites did not show the superparamagnetic characteristic. However, the Hc value was small enough for the redispersion of the mMSHCs after the magnetic separation, i.e., once the magnet is removed after the collection of our mMSHCs by the magnet as in Figure $6(\mathrm{~b})$, they were easily redispersed by slight shake.

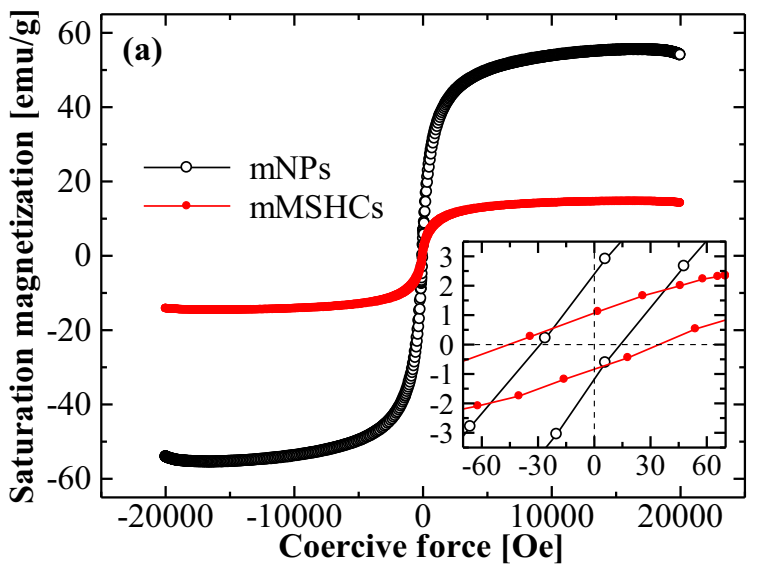

(b)

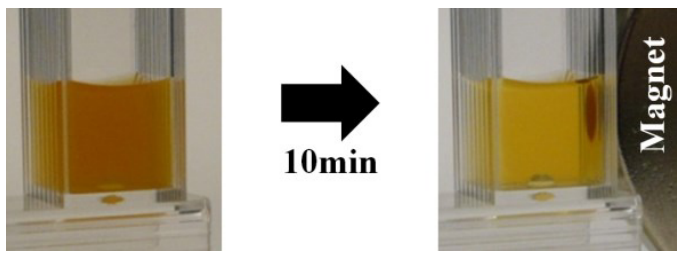

Fig. 6. (a) Magnetization curves of mNPs and mMSHCs. (b) Magnetic separation of mMSHCs using a neodymium magnet.

\section{Conclusions}

The method for synthesizing the mMSHCs was developed by combining our protocol for the MSHC synthesis with the LbL method to integrate the mNPs onto the PS particles, which act as the templates for the hollow cavity in the MSHC synthesis. The mNPs were successfully incorporated in the inner surface of the MSHC cavity. The structural characteristics of our conventional MSHCs, i.e., the uniformities of the size and shape in the capsules and the structure of the mesopores in their silica shells, were maintained in the resultant mMSHCs. The magnetic separation was also demonstrated. Because it had been demonstrated that the diameter of the MSHC could be reduced less than $100 \mathrm{~nm}$ [3], our mMSHCs could be one of the candidates for the magnetically targeted carriers.

\section{Acknowledgements}

The authors acknowledge the financial supports from The Institute of Science and Technology, Meiji University and partially from JSPS KAKENHI Grant Number 16K01399.

\section{References}

1. Y. Li, J. Shi, Adv. Mater. 26, 3176 (2014)

2. Y. Bao, C. Shi, T. Wang, X. Li, J. Ma, Micropor. Mesopor. Mater. 227, 121 (2016)

3. N. Kato, T. Ishii, S. Koumoto, Langmuir 26, 14334 (2010)

4. N. Kato, N. Kato, Micropor. Mesopor. Mater. 219, 230 (2016)

5. Y. Yamaguchi, N. Kato, TechConnect World 2014 Proceedings, in: Nanotechnology 2014, vol. 2, pp. 315-318 (CRC Press, Florida, 2014)

6. Y. Chen, H. Chen, J. Shi, Adv. Mater. 25, 3144 (2013)

7. P. Kovačík, M. Singh, F. Štěpánek, Chem. Eng. J. 232, 591 (2013)

8. J. Liu, Y. Deng, C. Liu, Z. Sun, D. Zhao, J. Colloid Interface Sci. 333, 329 (2009)

9. F. Wang, Y. Tang, B. Zhang, B. Chen, Y. Wang, J. Colloid Interface Sci. 386, 129 (2012)

10. J. Zhou, W. Wu, D. Caruntu, M.H. Yu, A. Martin, J.F. Chen, C.J. O'Connor, W.L. Zhou, J. Phys. Chem. C 111, 17473 (2007)

11. L. Zhang, S. Qiao, Y. Jin, Z. Chen, H. Gu, G.Q. Lu, Adv. Mater. 20, 805 (2008)

12. F. Liu, J. Wang, Q. Cao, H. Deng, G. Shao, D.Y.B. Deng, W. Zhou, Chem. Commun. 51, 2357 (2015)

13. W. Zhao, H. Chen, Y. Li, L. Li, M. Lang, J. Shi, Adv. Funct. Mater. 18, 2780 (2008)

14. Y. Zhu, E. Kockrick, T. Ikoma, N. Hanagata, S. Kaskel, Chem. Mater. 21, 2547 (2009)

15. Y. Zhu, Y. Fang, S. Kaskel, J. Phys. Chem. C 114, $16382(2010)$

16. X. Zhang, L. Clime, H. Roberge, F. Normandin, L'H. Yahia, E. Sacher, T. Veres, J. Phys. Chem. C 115, 1436 (2011)

17. C.X. Lin, Z. Li, S. Brumbley, L. Petrasovits, R. McQualter, C. Yu, G.Q. Lu, J. Mater. Chem. 21, 7565 (2011)

18. S. Sahu, N. Sinha, S.K. Bhutia, M. Majhi, S. Mohapatra, J. Mater. Chem. B 2, 3799 (2014)

19. F. Caruso, R.A. Caruso, H. Mohwald, Science 282, 1111 (1998)

20. F. Caruso, M. Spasova, A. Susha, M. Giersig, R.A. Caruso, Chem. Mater. 13, 109 (2001)

21. F. Caruso, Adv. Mater. 13, 11 (2001)

22. Y. Deng, W. Yang, C. Wang, S. Fu, Adv. Mater. 15, 1729 (2003)

23. I.W. Hamley, Introductrion to Soft Matter, Sec. 3.4 (John Wilay \& Sons, Ltd., West Sussex 2007)

24. D.W. Marquardt, J. Soc. Ind. Appl. Math. 11, 431 (1963)

25. A. Guinier, X-Ray Diffrction, Chap. 5 (Dover Publications Inc., New York, 1994) 